\title{
Evaluation of a New Interface Combining High-Flow Nasal Cannula and CPAP
}

\author{
Eugenio Garofalo, Andrea Bruni, Corrado Pelaia, Gianmaria Cammarota, Paolo Murabito, \\ Eugenio Biamonte, Karim Abdalla, Federico Longhini, and Paolo Navalesi
}

\begin{abstract}
BACKGROUND: This study assessed the effects of a new interface that combined CPAP $10 \mathrm{~cm} \mathrm{H}_{2} \mathrm{O}$ by using a helmet with high-flow nasal cannula (HFNC) at varying flows in healthy volunteers. Outcome measures included pharyngeal pressures, diaphragm kinetics, breathing frequency, the temperature inside the helmet, and comfort. METHODS: After baseline assessment during spontaneous breathing, HFNC was applied at flows of 30, 40, and $50 \mathrm{~L} / \mathrm{min}$. Successively, the volunteers underwent helmet CPAP at $10 \mathrm{~cm} \mathrm{H}_{2} \mathrm{O}$ and CPAP + HFNC at flows of 30, 40, and $50 \mathrm{~L} / \mathrm{min}$. We measured the variations of pharyngeal pressures at end-expiration and end-inspiration, referenced to spontaneous breathing for HFNC and to CPAP for CPAP + HFNC, diaphragm displacement and thickness at end-expiration and thickness at end-inspiration, breathing frequency, the temperature inside the helmet, the occurrence of the fog effect, and comfort. RESULTS: Variations of pharyngeal pressures at end-inspiration changes were small overall and clinically unimportant. With the mouth closed, at increasing HFNC flow, variations of pharyngeal pressures at endexpiration increased during both HFNC (from 2.8 up to 7.7) and, to a lesser extent, CPAP + HFNC (from 2.7 up to 3.8) $(P<.001$ for all comparisons). These variations were attenuated during open-mouth breathing. HFNC $\geq 40 \mathrm{~L} / \mathrm{min}$ and CPAP + HFNC $\geq 40 \mathrm{~L} / \mathrm{min}$ compared with spontaneous breathing and CPAP, respectively, increased diaphragm displacement $(P=.001)$, thickness at end-inspiration and thickness at end-expiration $(P<.003$ for both $)$. At all flows, breathing frequency was slightly, although significantly, lower with CPAP + HFNC than with HFNC alone $(P<.003)$. The temperature inside the helmet increased slightly and insignificantly at flows of $\leq 40 \mathrm{~L} / \mathrm{min}$ with CPAP + HFNC compared with CPAP alone. The fog effect never occurred, whereas comfort was always rated as optimal, without differences between trials. CONCLUSIONS: CPAP + HFNC was well tolerated, with no adverse effects. Based on our findings, there was no need to vary the CPAP level when adding HFNC. At least in healthy subjects, CPAP + HFNC at $30 \mathrm{~L} / \mathrm{min}$ seemed to be the best combination. Key words: continuous positive airway pressure; high-flow nasal cannula; pharyngeal pressure; diaphragm contractility; diaphragm ultrasound; comfort; breathing pattern; healthy volunteers. [Respir Care 2019;64(10):1231-1239. (C 2019
\end{abstract} Daedalus Enterprises]

\section{Introduction}

The primary supportive treatment in patients who are hypoxemic is oxygen therapy, which is commonly deliv-

Drs Garofalo, Bruni, Pelaia, Abdalla, Longhini, and Navalesi are affiliated with the Intensive Care Unit, University Hospital Mater Domini, Department of Medical and Surgical Sciences, Magna Graecia University, Catanzaro, Italy. Dr Cammarota is affiliated with the Anesthesia and Intensive Care, "Maggiore della Carità" Hospital, Novara, Italy. Dr Murabito is affiliated with the Department of Clinical and Experimental ered by using nasal prongs or masks. New devices have recently been made available to deliver oxygen via highflow nasal cannula (HFNC). HFNC delivers heated and

\footnotetext{
Medicine, University of Catania, Catania, Italy. Dr Biamonte is affiliated with the Intensive Care Unit, University Hospital Mater Domini, Catanzaro, Italy.
}

Intersurgical provided the prototype of the experimental device.

Drs Garofalo and Bruni are co-first authors. 
humidified gas up to $60 \mathrm{~L} / \mathrm{min}$, with an $\mathrm{F}_{\mathrm{IO}_{2}}$ that ranges from 0.21 to 1.0 via a wide-bore soft nasal prong. Warming and humidification of the inspired gas prevent the adverse effects of cool dry gases on the airway epithelium and facilitate expectoration. HFNC also washes out exhaled $\mathrm{CO}_{2}$ from the pharyngeal dead space. ${ }^{1} \mathrm{HFNC}$ has been shown to be an effective means to deliver oxygen therapy in many clinical conditions.

In healthy patients during spontaneous unassisted breathing, end-expiratory pharyngeal pressure is $\sim 0.3$ and $0.8 \mathrm{~cm} \mathrm{H}_{2} \mathrm{O}$ with opened and closed mouth, respectively. ${ }^{2}$ Compared with unassisted spontaneous breathing, HFNC generates greater pharyngeal pressure during expiration, whereas, in the course of inspiration, it drops to zero, ${ }^{1}$ which limits the effectiveness of HFNC in patients with lung edema and/or collapse. By recruiting atelectatic regions, ${ }^{3}$ reducing venous admixture, ${ }^{4}$ and decreasing the inspiratory effort, ${ }^{5}$ CPAP is likely more effective in these instances. ${ }^{6}$ Compared with noninvasive ventilation by application of inspiratory pressure support, CPAP offers several advantages, which include ease of use and lack of patient-ventilator asynchrony. ${ }^{7-11}$

CPAP may be applied either through a mask or a helmet. The latter is better tolerated than face masks and allows more continuous CPAP application. ${ }^{12,13}$ When applying CPAP by using a helmet, however, heating and humidification of the inhaled gas is problematic because of condensation of water inside the interface, the so-called fog effect. ${ }^{14}$ Moreover, in patients who receive CPAP via helmet, some rebreathing occurs. ${ }^{15}$ To overcome these limitations and combine the beneficial effects of HFNC and CPAP, we designed a new device that combines HFNC and helmet CPAP (patent in progress, European Patent application number EP20170199831).

Recently, Mauriusb et al ${ }^{16}$ found this combination capable of providing stable CPAP and effective $\mathrm{CO}_{2}$ wash-

\footnotetext{
Dr Navalesi discloses relationships with Maquet Critical Care, Draeger, Intersurgical, Orionpharma, Philips, Resmed, MSD, and Novartis. Dr Navalesi contributed to the development of the helmet Next, whose license for patent belongs to Intersurgical, and receives royalties for that invention. Drs Longhini and Navalesi contributed to the development of the device (CPAP + HFNC) discussed in the present study, whose patent is in progress (European Patent application number EP20170199831). The remaining authors have disclosed no conflicts of interest.

Supplementary material related to this paper is available at http:// www.rcjournal.com.

Correspondence: Federico Longhini MD, Intensive Care Unit, University Hospital Mater Domini Department of Medical and Surgical Sciences Magna Graecia University Viale Europa - Loc. Germaneto 88100, Catanzaro, Italy. E-mail: longhini.federico@gmail.com.
}

DOI: $10.4187 /$ respcare.06871

\section{QUICK LOOK}

\section{Current knowledge}

Devices that deliver heated and humidified gas via highflow nasal cannula (HFNC) at up to $60 \mathrm{~L} / \mathrm{min}$ have been shown to be an effective means to deliver oxygen therapy in many clinical conditions. As opposed to HFNC, CPAP provides positive airway pressure throughout the entire respiratory cycle by recruiting atelectatic regions, reducing venous admixture, and decreasing inspiratory effort.

\section{What this paper contributes to our knowledge}

Pharyngeal pressure changes were small overall and not clinically relevant during the combination of HFNC + CPAP. CPAP + HFNC was well tolerated overall, did not significantly alter the temperature inside the helmet, and did not cause any fog effect in comparison with CPAP only. HFNC at a flow of 30 $\mathrm{L} / \mathrm{min}$ with CPAP $10 \mathrm{~cm} \mathrm{H}_{2} \mathrm{O}$ seemed to be the best combination in healthy volunteers.

out from the upper airways with negligible $\mathrm{CO}_{2}$ rebreathing. Nonetheless, because of the complex interplay between CPAP and HFNC, the amount of truly applied airway pressure, diaphragm function, and temperature inside the helmet might be affected to some extent. We, therefore, designed this study to assess, in healthy volunteers, the effect of adding HFNC at varying flows to helmet CPAP set at $10 \mathrm{~cm} \mathrm{H}_{2} \mathrm{O}$.

\section{Methods}

The study was conducted from December 2017 to February 2018 in the IICU of the "Mater Domini" University Hospital, Catanzaro, Italy. The study was approved by the local ethics committee "Comitato Etico Sezione Area Centro-Regione Calabria" (protocol 178/2017) on July 20, 2017. Written informed consent was obtained from the volunteers for publication of their individual details and accompanying images in this manuscript. The trial has been prospectively registered at the Australian New Zealand Clinical Trials Registry (www.anzctr.org.au; trial ID ACTRN12617001526369). The authors will share all of the individual participant data collected during the trial after de-identification to researchers who provide a methodologically sound proposal.

\section{Subjects and Study Protocol}

We enrolled 14 healthy adult medical students ( 7 males and 7 females) naive to mechanical ventilation, with no 


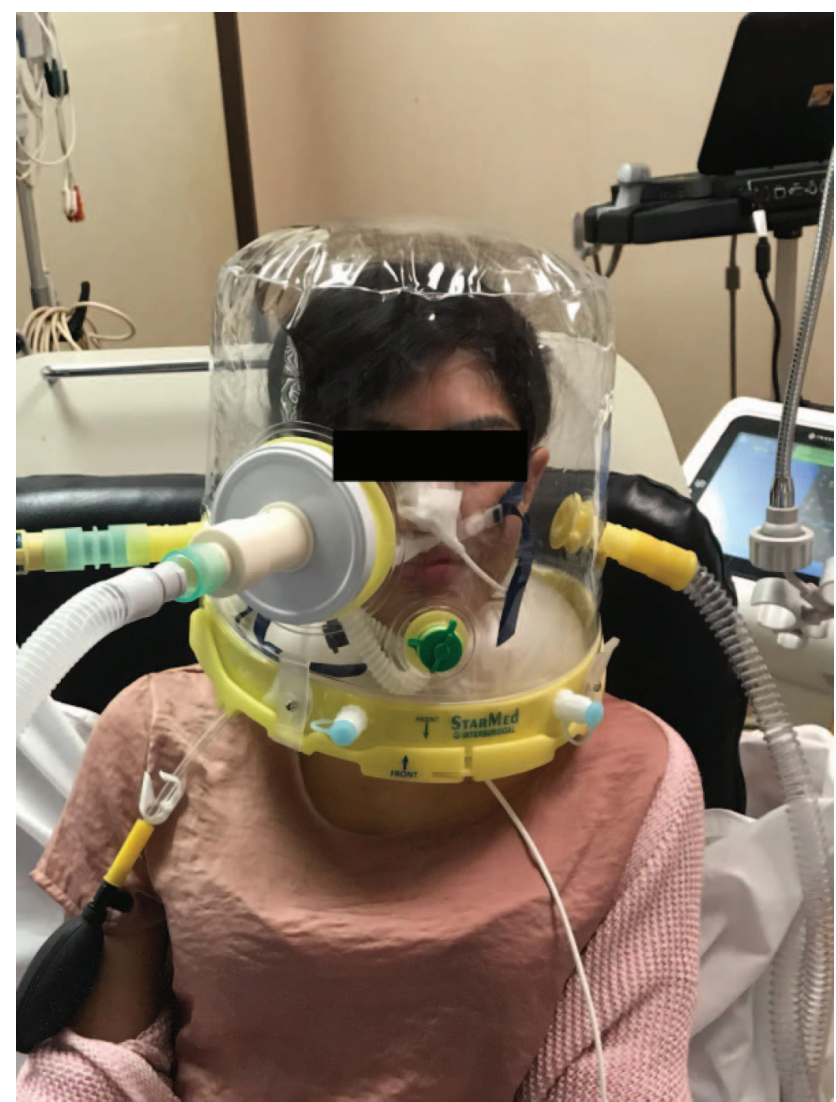

Fig. 1. A subject demonstrating the experimental setup of helmet CPAP + high-flow nasal cannula.

history of ear-nose-throat surgery or epistaxis in the previous 12 months. None of the volunteers either coauthored the study or was aware of the study purposes. The subjects maintained the semi-recumbent position throughout the whole study period. After anesthetizing the nostrils and pharynx with topical sprayed lidocaine, a dedicated manometry catheter with multiple side holes and occluded tip (BioEngineering Laboratories, Cantù, Italy) was positioned via the nose into the oropharynx, just below the uvula, under headlight visual inspection. The catheter was then connected to a calibrated pressure transducer (FluxMed GrE, MBMed, Buenos Aires, Argentina). The experimental setup during CPAP + HFNC in one representative subject is depicted in Figure 1. Data were displayed online throughout the study protocol for visual determination of the quality of the signal (FluxView, MBMed) and stored in a laptop computer for subsequent analysis (FluxReview, MBMed). At the end of each trial, and whenever necessary, the catheter was flushed with air injected through a 10 -mL syringe.

After baseline assessment during spontaneous breathing on room air, HFNC was applied by means of a dedicated device (AIRVO2, Fisher \& Paykel Healthcare, Auckland, New Zealand) at $31^{\circ} \mathrm{C} ; \mathrm{F}_{\mathrm{IO}_{2}} 0.21$; and flows of
30, 40, 50, and $60 \mathrm{~L} / \mathrm{min}$. Successively, the volunteers underwent CPAP, delivered through a helmet (Castar Next, Intersurgical, Mirandola, Italy), with an adjustable PEEP valve $\left(2.5-20 \mathrm{~cm} \mathrm{H}_{2} \mathrm{O}\right)$ set at $10 \mathrm{~cm} \mathrm{H}_{2} \mathrm{O}$ (Intersurgical). The helmet was connected to a turbine-driven ventilator (Monnal T60, Air Liquide Medical Systems, Antony, France) set to deliver room air at a continuous flow of $60 \mathrm{~L} / \mathrm{min}$. CPAP and HFNC were then combined by using with the same aforementioned settings. However, because it was not possible to achieve $60 \mathrm{~L} / \mathrm{min}$ through the nasal prongs, we limited our investigation to 30,40 , and 50 $\mathrm{L} / \mathrm{min}$. In all experimental conditions, the measurements were performed after $15 \mathrm{~min}$ to allow the subjects to become acquainted with the experimental equipment.

\section{Data Acquisition and Analysis}

We acquired pharyngeal pressures with the mouth either opened or closed, and recorded the last minute of each trial for off-line analysis. From the recorded tracings, we determined pharyngeal pressures at end-expiration and end-inspiration, and breathing frequency. We, therefore, computed the variations of pharyngeal pressures at end-expiration and at end-inspiration, as referenced to spontaneous breathing for HFNC and to CPAP for CPAP + HFNC trials.

At the end of each trial, 2 investigators (GC and $\mathrm{CP}$ ) unaware of the study aims independently performed bedside sonographic evaluation of the right hemi-diaphragm, as previously described. ${ }^{17,18}$ The probe placement site was marked by the first observer (GC). ${ }^{18}$ Briefly, we measured diaphragm displacement through a $3.5-5 \mathrm{MHz}$ phasedarray probe, placed immediately below the costal margin in the mid clavicular line and directed medially, cephalad, and dorsally, so that the ultrasound beam reached perpendicularly across the posterior third of the hemi-diaphragm. The M-mode was used to display the motion of the anatomic structures along the selected line and to assess the diaphragm displacement. To assess diaphragm thickness, we placed a linear 13-MHz probe at the 9th to 10th intercostal space, close to the mid axillary line, to identify the apposition zone of the diaphragm. Diaphragmatic thickness was determined in the M-mode at end-expiration and peak inspiration (thickness at end-inspiration) as the distance between the diaphragmatic pleura and the peritoneum. ${ }^{17,18}$ At every protocol step, each investigator independently performed and recorded 3 sets of measurements. The 6 measurements were then averaged.

To ascertain whether incremental flow of humidified air would increase the temperature inside the helmet, we assessed room temperature by positioning a precision probe thermometer at $4-5 \mathrm{~cm}$ from the subject's cheek, as reference during spontaneous breathing and HFNC, and inside the helmet during CPAP and CPAP + HFNC. We 
also assessed the occurrence of the fog effect by means of an arbitrary scale ( 0 , no fog at all; 1 , presence of vapor, 2 , water drops), evaluated by two observers (GC and $\mathrm{CP}$ ) blind to the study aim. Finally, an 11-point numeric rating scale, including numbers and descriptors, was used to assess the subject's comfort, as previously reported. ${ }^{11,19}$ After an explanation of the rating scale before study protocol initiation, each subject was asked to score his or her comfort by indicating a number between 0 (worst possible comfort) and 10 (best possible comfort). The obtained scores were recorded without any additional indications or comments. ${ }^{11,19}$

\section{Statistical Analysis}

Because no proper sample-size calculation was possible due to the lack of previously published data, we arbitrarily considered 14 subjects sufficient for our purposes. However, we conducted a post hoc paired $t$ test power analysis. Given a mean difference between CPAP and CPAP + HFNC of $30 \mathrm{~L} / \mathrm{min}$ in variations in pharyngeal pressures at end-expiration at opened mouth of $1.1 \mathrm{~cm} \mathrm{H}_{2} \mathrm{O}$, a standard deviation difference of 0.6 and a $\alpha$-error of 0.05 , and, when considering our total sample size $(N=14)$, we had an actual power equal to 1 for this variable in its most conservative condition.

To evaluate the consistency of sonographic assessments by different investigators, we used the interclass correlation coefficient. Data are presented as median (25-75\% interquartile range). The analysis of variance on ranks for repeated measures (Friedman test) was used to compare continuous variables. Pairwise multiple comparisons were conducted with the Wilcoxon rank-sum test, with adjusting the threshold for statistical significance by means of Bonferroni correction for multiple comparisons. In particular, we compared the following: (1) HFNC versus spontaneous breathing, (2) CPAP + HFNC versus CPAP, (3) CPAP versus spontaneous breathing, and (4) CPAP + HFNC versus HFNC at the same flow. We applied the Bonferroni correction for multiple comparisons to guarantee a type- 1 error probability of 0.05 over all tests performed on each response variable. Because 10 different tests were conducted for each variable, $P<.005$ was considered significant.

\section{Results}

The mean \pm SD age of participants was $24.5 \pm 1.6 \mathrm{y}$, weight was $61 \pm 11 \mathrm{~kg}$, height $172 \pm 10 \mathrm{~cm}$, whereas the body mass index was $21.3 \pm 1.8 \mathrm{~kg} / \mathrm{m}^{2}$. No subject asked to discontinue the study protocol.

\section{Airway (Pharyngeal) Pressure}

Depicted in Figure 2 are all the conditions under evaluation, variation of variations of pharyngeal pressures at end-inspiration and at end-expiration in the upper and lower panels, respectively, while breathing with opened (left panels) and closed (right panels) mouth. Detailed data are reported in online supplementary materials (see the supplementary materials at http://www.rcjournal.com). Overall, compared with HFNC, CPAP + HFNC was characterized by lower variations of pharyngeal pressures at end-inspiration and at end-expiration at corresponding flow. Both variations of pharyngeal pressures at end-inspiration and pharyngeal pressures at end-expiration significantly increased with HFNC and CPAP + HFNC compared with spontaneous breathing and CPAP, respectively. However, the changes observed for variation of pharyngeal pressures at end-inspiration were overall small, and achieved their maximum at $50 \mathrm{~L} / \mathrm{min}$ with the mouth closed (1.1 [1.01.2] $\mathrm{cm} \mathrm{H}_{2} \mathrm{O}$ and 0.4 [0.3-0.9] $\mathrm{cm} \mathrm{H}_{2} \mathrm{O}$, with HFNC and CPAP + HFNC, respectively). The variation of pharyngeal pressures at end-expiration was definitely higher during both HFNC (from 2.8 up to $7.7 \mathrm{~cm} \mathrm{H}_{2} \mathrm{O}$ ) and CPAP + HFNC (from 2.7 up to $3.8 \mathrm{~cm} \mathrm{H}_{2} \mathrm{O}$ ) while breathing with a closed mouth. Such variations were less relevant during opened mouth breathing (from 0.9 up to $2.3 \mathrm{~cm} \mathrm{H}_{2} \mathrm{O}$ and from 1.1 up to $1.9 \mathrm{~cm} \mathrm{H}_{2} \mathrm{O}$, for HFNC and CPAP + HFNC, respectively).

\section{Diaphragm Ultrasound and Breathing Frequency}

The interclass correlation coefficients were 0.98 , 95\% CI 0.97-0.99 for both diaphragm displacement and thickness at end-inspiration assessments, and were 0.96, 95\% CI 0.95-0.97 for thickness at end-expiration. As displayed in Table 1, compared with spontaneous breathing, both HFNC and CPAP + HFNC significantly increased diaphragm displacement at flow $\geq 40 \mathrm{~L} / \mathrm{min}(P=.001 \mathrm{for}$ all comparisons). In addition, CPAP and CPAP + HFNC $30 \mathrm{~L} / \mathrm{min}$ were characterized by higher diaphragm displacement when compared with spontaneous breathing $(P=.001)$ and HFNC $30 \mathrm{~L} / \mathrm{min}(P<.001)$, respectively. Compared with spontaneous breathing, both thickness at end-inspiration and thickness at end-expiration significantly increased during HFNC $40 \mathrm{~L} / \mathrm{min}$ and HFNC $50 \mathrm{~L} / \mathrm{min}$. Compared with CPAP, CPAP + HFNC $40 \mathrm{~L} / \mathrm{min}$ significantly augmented both thickness at endinspiration and thickness at end-expiration, with CPAP + HFNC $50 \mathrm{~L} / \mathrm{min}$ only thickness at end-inspiration. Furthermore, thickness at end-inspiration was significantly higher during CPAP (compared with spontaneous breathing) and CPAP + HFNC $30 \mathrm{~L} / \mathrm{min}$ (compared with HFNC 30 $\mathrm{L} / \mathrm{min}$ ); no significant differences in thickness at end-expiration were observed between CPAP and spontaneous 

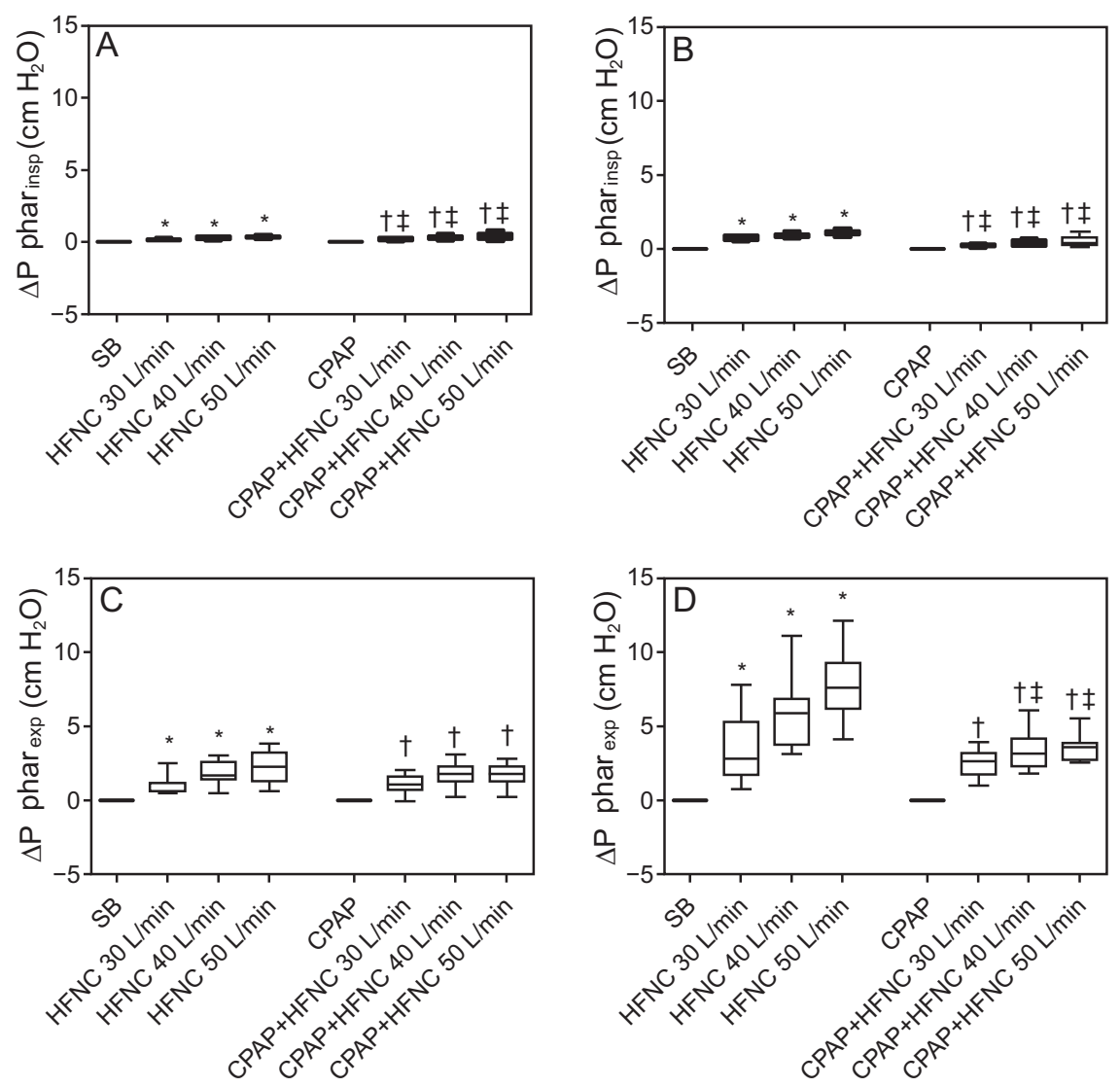

Fig. 2. Median variations of pharyngeal pressures at end-inspiration ( $A$ and $B)$ and end-expiration $(C$ and $D)$ while breathing with the mouth opened $(A$ and $C)$ and the mouth closed $(B$ and $D)$ for all conditions under evaluation. Boxes indicate 25th and 75th percentiles, horizontal lines show the median, and whiskers represent the 10th and 90 th percentile. ${ }^{*} P<.005$ compared with spontaneous breathing. $\dagger P<.005$ compared with CPAP; $\ddagger P<.005$ between HFNC and CPAP + HFNC at the same flow. SB = spontaneous breathing, HFNC = high-flow nasal cannula, phar $_{\text {insp }}=$ pharyngeal end-inspiration, phar $_{\text {exp }}=$ pharyngeal end-expiration.

breathing, and between CPAP + HFNC and HFNC at the same flow.

Also shown in Table 1, breathing frequency was slightly, although significantly, lower with CPAP compared with spontaneous breathing and CPAP + HFNC as opposed to HFNC at the corresponding flow. Compared with spontaneous breathing, HFNC flow $\geq 40 \mathrm{~L} / \mathrm{min}$ decreased breathing frequency, whereas, compared with CPAP, only CPAP + HFNC $50 \mathrm{~L} / \mathrm{min}$ significantly decreased breathing frequency.

\section{Temperature, Comfort, and Fog Effect}

The median (interquartile range) temperature assessed inside the interface during CPAP $\left(27[27-28]{ }^{\circ} \mathrm{C}\right)$, $\mathrm{CPAP}+\mathrm{HFNC} 30 \mathrm{~L} / \mathrm{min}\left(28\right.$ [27-28] $\left.{ }^{\circ} \mathrm{C}\right)$, $\mathrm{CPAP}+\mathrm{HFNC} 40 \mathrm{~L} / \mathrm{min}\left(28\right.$ [28-29] $\left.{ }^{\circ} \mathrm{C}\right)$, and CPAP + HFNC $50 \mathrm{~L} / \mathrm{min}\left(29[28-30]{ }^{\circ} \mathrm{C}\right)$ were all significantly higher compared with room temperature (24 [23-25] $\left.{ }^{\circ} \mathrm{C}\right)(P<.001$ for all comparisons $)$. The addition of heated humidified flow through the nasal cannula slightly, although significantly, increased the temperature only at CPAP + HFNC $50 \mathrm{~L} / \mathrm{min}$ compared with CPAP $(P=.004)$.

Despite the increased temperature that occurred during CPAP and CPAP + HFNC, all the subjects reported optimal comfort during all the tested conditions. In particular, the numeric rating scale was only slightly, although significantly, reduced during HFNC $50 \mathrm{~L} / \mathrm{min}$ compared with spontaneous breathing $(P=.003)$. No differences were reported between (1) CPAP and CPAP + HFNC at all flows, (2) CPAP and spontaneous breathing, and (3) CPAP + HFNC and HFNC at the same flow (Table 1). No subject reported discomfort related to excessive air temperature, whereas the fog effect was scored as $0(0-0)$ in all conditions (not displayed in Table 1).

\section{Discussion}

In healthy volunteers unaware of the study purposes, we found that adding HFNC to CPAP (compared to CPAP) (1) did not importantly alter either the preset airway pres- 


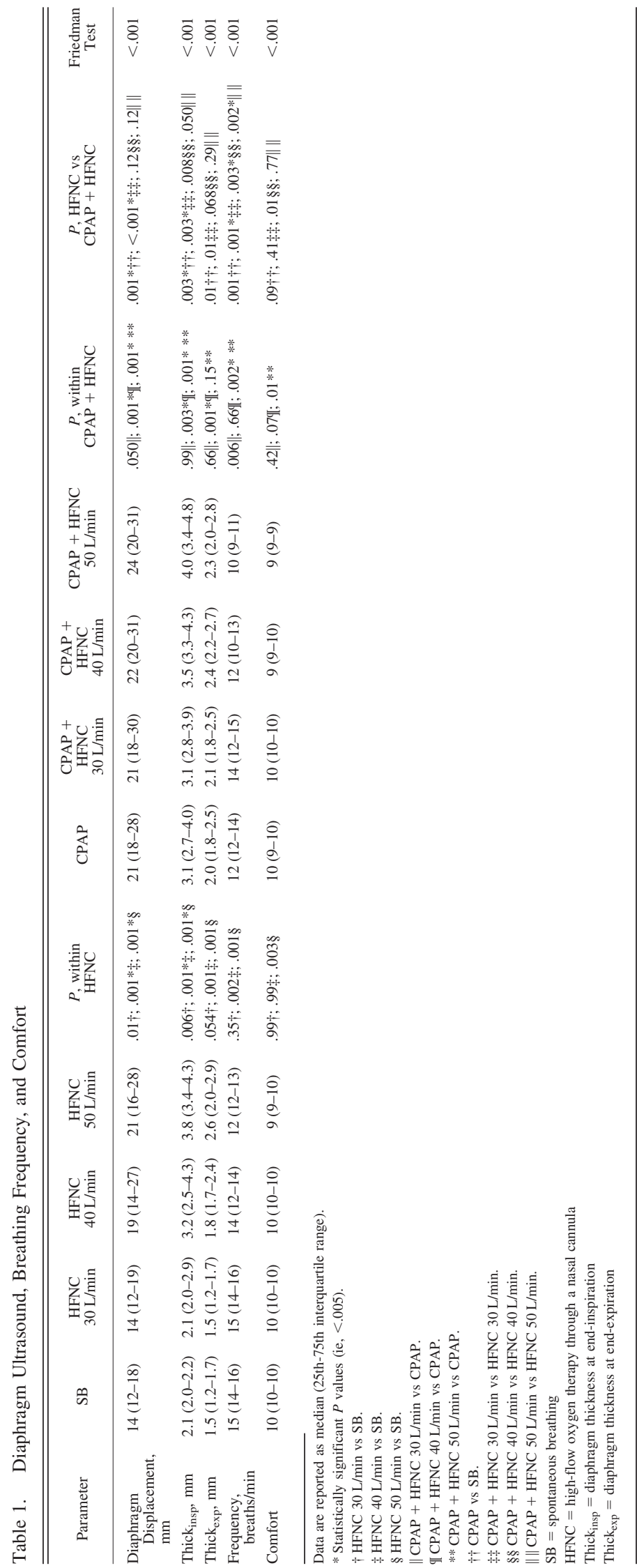


sure during inspiration or the temperature inside the helmet, (2) increased expiratory airway pressure proportionally to the flow administered by HFNC but to a lower extent than HFNC alone (as compared to spontaneous breathing), (3) produced only slight modifications of thickness at end-inspiration and thickness at end-expiration compared with CPAP alone, (4) did not cause the fog effect, and (5) did not worsen comfort.

In patients with acute hypoxemic respiratory failure due to pulmonary consolidation of different etiologies, CPAP increases end-expiratory lung volume by promoting alveolar recruitment, ${ }^{15}$ reduces venous admixture ${ }^{4}$ and the work of breathing, ${ }^{5}$ and improves oxygenation faster and in a greater proportion of patients as opposed to standard oxygen therapy. ${ }^{20,21}$ However, poor mask fitting, pressure sores, and air leaks may cause discomfort and limit continuous and prolonged delivery of CPAP via a mask. The helmet eliminates these drawbacks, while guaranteeing optimal CPAP application. ${ }^{12,15}$ However, adequate conditioning of the inspired gas is problematic during CPAP via a helmet, ${ }^{14}$ which may represent a major problem in patients with copious secretions.

Mauri et al ${ }^{16}$ recently investigated the combination of 3,5 , and $8 \mathrm{~cm} \mathrm{H}_{2} \mathrm{O}$ and HFNC set at $50 \mathrm{~L} / \mathrm{min}$ in healthy volunteers. They measured the mean airway pressure within the CPAP + HFNC system and found that it closely corresponded to the set PEEP level with small airway pressure oscillations during the respiratory cycle. ${ }^{16}$ Furthermore, they found that the inspiratory $\mathrm{CO}_{2}$ pressure measured at the hypopharynx was significantly higher with the helmet alone as opposed to both HFNC and CPAP + HFNC. ${ }^{16}$ Finally, compared with CPAP alone, CPAP + HFNC decreased breathing frequency, which was predominantly attributed to upper-airway washout reduced $\mathrm{CO}_{2}$ rebreathing. ${ }^{16}$

Our results corroborated and enhanced the notion that this approach was feasible, well tolerated, and with no adverse effects. In keeping with Mauri et al, ${ }^{16}$ compared with CPAP alone, we found that adding HFNC to helmet CPAP reduced breathing frequency. Also, combining helmet and HFNC did not worsen subject comfort compared with both CPAP and HFNC alone. In addition, we showed that, irrespective of HFNC flow, the fog effect never occurred. ${ }^{14}$ We also measured the temperature inside the helmet in all experimental conditions because previous work conducted on 28 healthy subjects during helmet CPAP showed that increasing temperature worsens comfort. ${ }^{22}$

Nevertheless, it remained fundamental to determine whether and to what extent the interplay between CPAP and HFNC would alter the amount of pressure applied by CPAP. We reasoned that measuring pressure within the helmet would not provide sufficient data to answer these crucial questions and, therefore, chose to measure airway pressure within the pharynx in all experimental conditions.
HFNC + CPAP increased expiratory airway pressure proportionally to the flow administered but to a lower extent than HFNC alone. These differences were less notable when the subjects breathed with an opened mouth, as generally happens in patients with respiratory distress and dyspnea. More important, regardless of mouth aperture and HFNC flow, airway pressure was unaffected during inspiration.

We also investigated diaphragm kinetics by ultrasound. In healthy individuals, CPAP induces hyperinflation, which affects the length of diaphragm fibers and muscle kinetics. ${ }^{23-25}$ In keeping with the results of a previous study, ${ }^{23}$ we observed increments of $25 \%$ and $45 \%$ for thickness at end-expiration and thickness at end-inspiration, respectively, after application of CPAP $10 \mathrm{~cm} \mathrm{H}_{2} \mathrm{O}$. Further increments of both thickness at end-expiration and thickness at end-inspiration compared with CPAP were observed with CPAP + HFNC $40 \mathrm{~L} / \mathrm{min}$ and $50 \mathrm{~L} / \mathrm{min}$, although not $30 \mathrm{~L} / \mathrm{min}$.

Overall, the $30 \mathrm{~L} / \mathrm{min}$ flow through the nasal cannula during CPAP + HFNC did not produce major and clinically relevant modifications of the pharyngeal pressure compared with CPAP alone. Moreover, this flow was well tolerated, without altering the temperature inside the helmet or producing a fog effect. Noteworthy, in a population of subjects with hypoxemic acute respiratory failure, most of the effects of HFNC on respiratory effort and upper airways washout were already obtained at $30 \mathrm{~L} / \mathrm{min}^{26}$ Nonetheless, we could not exclude the possibility that higher flows may provide better results in patients who are acutely ill; further studies are necessary to evaluate this device in various patient populations.

Our study had some limitations that deserve discussion. First, for the purpose of assessing feasibility, we conducted our investigation on healthy individuals, which made our findings difficult to generalize. We share this limitation with several studies that evaluated new devices. ${ }^{16,27-29}$ Furthermore, a bench assessment in these experimental conditions would not be possible. ${ }^{30,31}$ Second, because we performed a short-term evaluation, we could not exclude that the observed results would be different after longer periods of application. This holds particularly true for comfort assessment. Again, we also share this limitation with the majority of studies that evaluated new devices, both in healthy volunteers ${ }^{16,27}$ and in patients..$^{11,19,32}$ Third, we did not measure breathing pattern and inspiratory effort. Because an external apparatus, such as a mouthpiece and esophageal catheter, would affect the breathing pattern and comfort, ${ }^{33,34}$ we preferred to adopt a noninvasive, although reproducible, technique such as diaphragm ultrasound. ${ }^{17}$

\section{Conclusions}

Compared with CPAP only, CPAP + HFNC was well tolerated, did not significantly alter the temperature inside 
the helmet, and did not cause any fog effect. Notable, because the addition of HFNC did not affect airway pressure during inspiration, the CPAP level should not be modified with this new device. In healthy volunteers, compared with CPAP alone, adding heated humidified air through a nasal cannula at a flow of $30 \mathrm{~L} / \mathrm{min}$ did not significantly alter pharyngeal pressure throughout the respiratory cycle, did not affect diaphragm kinetics, and was very well tolerated. Further studies are deemed necessary to confirm these observations in patients who are critically ill and to evaluate the potential clinical benefits.

\section{REFERENCES}

1. Renda T, Corrado A, Iskandar G, Pelaia G, Abdalla K, Navalesi P. High-flow nasal oxygen therapy in intensive care and anaesthesia. Br J Anaesth 2018;120(1):18-27.

2. Groves N, Tobin A. High flow nasal oxygen generates positive airway pressure in adult volunteers. Aust Crit Care 2007;20(4):126-131.

3. Cammarota G, Vaschetto R, Turucz E, Dellapiazza F, Colombo D, Blando C, et al. Influence of lung collapse distribution on the physiologic response to recruitment maneuvers during noninvasive continuous positive airway pressure. Intensive Care Med 2011;37(7): 1095-1102.

4. Lindner KH, Lotz P, Ahnefeld FW. Continuous positive airway pressure effect on functional residual capacity, vital capacity and its subdivisions. Chest 1987;92(1):66-70.

5. Lenique F, Habis M, Lofaso F, Dubois-Randé JL, Harf A, Brochard L. Ventilatory and hemodynamic effects of continuous positive airway pressure in left heart failure. Am J Respir Crit Care Med 1997; 155(2):500-505.

6. Bellani G, Patroniti N, Greco M, Foti G, Pesenti A. The use of helmets to deliver non-invasive continuous positive airway pressure in hypoxemic acute respiratory failure. Minerva Anestesiol 2008; 74(11):651-656.

7. Nava $S$, Navalesi $P$, Carlucci A. Non-invasive ventilation. Minerva Anestesiol 2009;75(1-2):31-36.

8. Longhini F, Colombo D, Pisani L, Idone F, Chun P, Doorduin J, et al. Efficacy of ventilator waveform observation for detection of patient-ventilator asynchrony during NIV: a multicentre study. ERJ Open Res 2017;3(4). pii: 00075-2017.

9. Garofalo E, Bruni A, Pelaia C, Liparota L, Lombardo N, Longhin F, Navalesi P. Recognizing, quantifying and managing patient-ventilator asynchrony in invasive and noninvasive ventilation. Expert Rev Respir Med 2018;12(7):557-567.

10. Cammarota G, Olivieri C, Costa R, Vaschetto R, Colombo D, Turucz $\mathrm{E}$, et al. Noninvasive ventilation through a helmet in postextubation hypoxemic patients: physiologic comparison between neurally adjusted ventilatory assist and pressure support ventilation. Intensive Care Med 2011;37(12):1943-1950.

11. Longhini F, Pan C, Xie J, Cammarota G, Bruni A, Garofalo E, et al. New setting of neurally adjusted ventilatory assist for noninvasive ventilation by facial mask: a physiologic study. Crit Care 2017; 21(1): 170 .

12. Principi T, Pantanetti S, Catani F, Elisei D, Gabbanelli V, Pelaia P, Leoni P. Noninvasive continuous positive airway pressure delivered by helmet in hematological malignancy patients with hypoxemic acute respiratory failure. Intensive Care Med 2004;30(1):147-150.

13. Tonnelier JM, Prat G, Nowak E, Goetghebeur D, Renault A, Boles JM, L'her E. Noninvasive continuous positive airway pressure ventilation using a new helmet interface: a case-control prospective pilot study. Intensive Care Med 2003;29(11):2077-2080.
14. Nava S, Navalesi P, Gregoretti C. Interfaces and humidification for noninvasive mechanical ventilation. Respir Care 2009;54(1):71-84.

15. Patroniti N, Foti G, Manfio A, Coppo A, Bellani G, Pesenti A. Head helmet versus face mask for non-invasive continuous positive airway pressure: a physiological study. Intensive Care Med 2003;29(10): 1680-1687.

16. Mauri T, Spinelli E, Mariani M, Guzzardella A, Del Prete C, Carlesso E, et al. Nasal high flow delivered within the helmet: a new non-invasive respiratory support. Am J Respir Crit Care Med 2019; 199(1):115-117.

17. Matamis D, Soilemezi E, Tsagourias M, Akoumianaki E, Dimassi S, Boroli F, et al. Sonographic evaluation of the diaphragm in critically ill patients. Technique and clinical applications. Intensive Care Med 2013;39(5):801-810.

18. Goligher EC, Laghi F, Detsky ME, Farias P, Murray A, Brace D, et al. Measuring diaphragm thickness with ultrasound in mechanically ventilated patients: feasibility, reproducibility and validity. Intensive Care Med 2015;41(4):734.

19. Cammarota G, Longhini F, Perucca R, Ronco C, Colombo D, Messina A, et al. New setting of neurally adjusted ventilatory assist during noninvasive ventilation through a helmet. Anesthesiology 2016;125(6):1181-1189.

20. L'Her E, Deye N, Lellouche F, Taille S, Demoule A, Fraticelli A, et al. Physiologic effects of noninvasive ventilation during acute lung injury. Am J Respir Crit Care Med 2005;172(9):1112-1118.

21. Cosentini R, Brambilla AM, Aliberti S, Bignamini A, Nava S, Maffei A, et al. Helmet continuous positive airway pressure vs oxygen therapy to improve oxygenation in community-acquired pneumonia: a randomized, controlled trial. Chest 2010;138(1):114-120.

22. Ueta K, Tomita T, Uchiyama A, Ohta N, Iguchi N, Goto Y, Fujino $\mathrm{Y}$. Influence of humidification on comfort during noninvasive ventilation with a helmet. Respir Care 2013;58(5):798-804.

23. Soilemezi E, Koco E, Tsimpos C, Tsagourias M, Savvidou S, Matamis D. Effects of continuous positive airway pressure on diaphragmatic kinetics and breathing pattern in healthy individuals. Respirology 2016;21(7):1262-1269.

24. Torres A, Kacmarek RM, Kimball WR, Qvist J, Stanek K, Whyte R, Zapol WM. Regional diaphragmatic length and EMG activity during inspiratory pressure support and CPAP in awake sheep. J Appl Physiol (1985) 1993;74(2):695-703.

25. Decramer M. Hyperinflation and respiratory muscle interaction. Eur Respir J 1997;10(4):934-941.

26. Mauri T, Alban L, Turrini C, Cambiaghi B, Carlesso E, Taccone P, et al. Optimum support by high-flow nasal cannula in acute hypoxemic respiratory failure: effects of increasing flow rates. Intensive Care Med 2017;43(10):1453-1463.

27. Vaschetto R, De Jong A, Conseil M, Galia F, Mahul M, Coisel Y, et al. Comparative evaluation of three interfaces for non-invasive ventilation: a randomized cross-over design physiologic study on healthy volunteers. Crit Care 2014;18(1):R2.

28. Costa R, Navalesi P, Antonelli M, Cavaliere F, Craba A, Proietti R, Conti G. Physiologic evaluation of different levels of assistance during noninvasive ventilation delivered through a helmet. Chest 2005; 128(4):2984-2990.

29. Racca F, Appendini L, Gregoretti C, Stra E, Patessio A, Donner CF, Ranieri VM. Effectiveness of mask and helmet interfaces to deliver noninvasive ventilation in a human model of resistive breathing. J Appl Physiol (1985) 2005;99(4):1262-1271.

30. Olivieri C, Costa R, Conti G, Navalesi P. Bench studies evaluating devices for non-invasive ventilation: critical analysis and future perspectives. Intensive Care Med 2012;38(1):160-167.

31. Olivieri C, Costa R, Spinazzola G, Ferrone G, Longhini F, Cammarota $\mathrm{G}$, et al. Bench comparative evaluation of a new generation and 


\section{Evaluation of a Combined Noninvasive InTERface}

standard helmet for delivering non-invasive ventilation. Intensive Care Med 2013;39(4):734-738.

32. Olivieri C, Longhini F, Cena T, Cammarota G, Vaschetto R, Messina A, et al. New versus conventional helmet for delivering noninvasive ventilation: a physiologic, crossover randomized study in critically ill patients. Anesthesiology 2016;124(1):101-108.
33. Perez W, Tobin MJ. Separation of factors responsible for change in breathing pattern induced by instrumentation. J Appl Physiol (1985) 1985;59(5):1515-1520

34. Weissman C, Askanazi J, Milic-Emili J, Kinney JM. Effect of respiratory apparatus on respiration. J Appl Physiol Respir Environ Exerc Physiol 1984;57(2):475-480. 